\title{
WEAK TYPE ESTIMATES FOR BOCHNER-RIESZ SPHERICAL SUMMATION MULTIPLIERS
}

BY

\author{
SAGUN CHANILLO $^{1}$ AND BENJAMIN MUCKENHOUPT ${ }^{2}$
}

ABStRACT. We consider the Bochner-Riesz multiplier

$$
\widehat{T_{\delta} f}(\xi)=\left(1-|\xi|^{2}\right)_{+}^{\delta} \hat{f}(\xi), \quad \delta>0
$$

where denotes the Fourier transform. It is shown that the multiplier operator $T_{\delta}$ is weak type $\left(p_{0}, p_{0}\right)$ acting on $L^{p_{0}}\left(\mathbf{R}^{n}\right)$ radial functions, where $p_{0}$ is the critical value $2 n /(n+1+2 \delta)$.

1. Introduction. Let $\hat{f}(\xi)$ denote the Fourier transform of the function $f(x)$. Let $B=\{\xi:|\xi| \leq 1\}$, the unit ball in $\mathbf{R}^{n}$. For $\delta>0$, define the multiplier operator

$$
\widehat{T_{\delta} f}(\xi)=\left(1-|\xi|^{2}\right)^{\delta} \chi_{B}(\xi) \hat{f}(\xi) .
$$

It is well known that these multiplier operators are unbounded on $L^{p}\left(\mathbf{R}^{n}\right)$ when $p \leq 2 n /(n+1+2 \delta)$ or $p \geq 2 n /(n-1-2 \delta)$ for $0<\delta \leq(n-1) / 2$. When $\delta>(n-1) / 2$ the convolution kernel of $T_{\delta}$, which is given by $J_{n / 2+\delta}(|x|)|x|^{-n / 2-\delta} \in L^{1}\left(\mathbf{R}^{n}\right)$, and so $T_{\delta}$ is bounded on $L^{p}$ for $1 \leq p \leq \infty$. Here $J_{\alpha}(|x|)$ denotes the Bessel function of order $\alpha$.

When $n=2$, it is known that when $0<\delta \leq \frac{1}{2}, T_{\delta}$ is bounded on $L^{p}\left(\mathbf{R}^{2}\right)$ for $4 /(3+2 \delta)<p<4 /(1-2 \delta)$. A proof of this result known as the multiplier theorem of Carleson-Sjölin, C. Fefferman and Hörmander may be found in $[\mathbf{3}]$. Results for $n>2$ may be found in $[\mathbf{1}, \mathbf{4}, \mathbf{5}, \mathbf{8}$ and $\mathbf{1 0}]$.

We prove here

THEOREM 1. Let $f$ be radial. Then for $\lambda>0,0<\delta \leq(n-1) / 2$ and $n \geq 2$,

$$
\left|\left\{x \in \mathbf{R}^{n}:\left|T_{\delta} f(x)\right|>\lambda\right\}\right| \leq C \lambda^{-p_{0}}\|f\|_{p_{0}}^{p_{0}}
$$

with $p_{0}=2 n /(n+1+2 \delta)$. The constant $C$ does not depend on $\lambda$ or $f$.

The case $\delta=0$ in Theorem 1 is considered in [5-7 and 2]. Using the interpolation theorem of Marcinkiewicz and duality the next theorem follows by considering the space of functions $\left(\int_{0}^{\infty}|f(r)|^{p} r^{n-1} d r\right)^{1 / p}$.

THEOREM 2. Let $f$ be radial. Then for $0<\delta \leq(n-1) / 2$ and $2 n /(n+1+2 \delta)<$ $p<2 n /(n-1-2 \delta)$,

$$
\left\|T_{\delta} f\right\|_{p} \leq c_{p}\|f\|_{p}
$$

As pointed out, we obtain Theorem 2 by interpolating the trivial estimate $\left\|T_{\delta} f\right\|_{2} \leq c\|f\|_{2}$ with that of Theorem 1 . Theorem 2 is not new and in fact another

Received by the editors April 24, 1985.

1980 Mathematics Subject Classification. Primary 42B20, 42B15, 44A15.

${ }^{1}$ Supported in part by N.S.F. grant MCS-8108814(A03).

2 Supported in part by N.S.F. grant MCS83-01481-01. 
way to obtain it is to use the complex interpolation theorem of E. Stein, the result of Herz [6] for $\delta=0$ and the $L^{1}$ result for $\delta>(n-1) / 2$.

We point out that the motivation for Theorem 1 comes from restriction phenomena for the Fourier transform. In fact for $f \in C_{0}^{\infty}\left(\mathbf{R}^{n}\right)$ and $|x| \rightarrow \infty$,

$$
\left|T_{\delta} f(x)\right| \sim c|\hat{f}(x /|x|)| /|x|^{-(n+1+2 \delta) / 2} .
$$

But for radial $f$,

$$
\|\hat{f}\|_{L^{\infty}\left(S^{n-1}\right)} \leq c\|f\|_{p_{0}}, \quad p_{0}=2 n /(n+1+2 \delta)
$$

Thus, we expect a weak type result.

There are natural analogues of Theorem 1 for Cesaro means of series of the orthogonal polynomials like the Gegenbauer polynomials. Weak type results can be proved at an appropriate critical value for the partial sum operator associated to Cesaro means of some order. These results will be taken up elsewhere.

We use the standard notation for the Hardy-Littlewood maximum function, i.e., for $x \in \mathbf{R}$,

$$
f^{*}(x)=\sup _{h>0} \frac{1}{2 h} \int_{x-h}^{x+h}|f(t)| d t .
$$

2. We will now prove the preliminary lemmas from which it will be possible to deduce Theorem 1. To do so we need some properties of Bessel functions. These may be found in $[\mathbf{1 1}]$.

LEMma (2.1). Let $J_{\alpha}(u)$ denote the Bessel function of order $\alpha$. Then for $s, u>0$ and $\alpha \geq 0$,

$$
\begin{aligned}
& \text { (a) } \frac{d}{d u}\left(J_{\alpha+1}(s u)\right)=s J_{\alpha}(s u)-\frac{\alpha+1}{u} J_{\alpha+1}(s u), \\
& \text { (b) } \frac{d}{d u}\left(J_{\alpha}(s u)\right)=-s J_{\alpha+1}(s u)+\frac{\alpha}{u} J_{\alpha}(s u), \\
& \text { (c) }\left|J_{\alpha}(s u)\right| \leq c(1+s u)^{-1 / 2} .
\end{aligned}
$$

LEMMA (2.2). For $\delta$ real, $0 \leq s \leq r-2$, any integer $j \geq 1$ and $\alpha, \beta \geq 0$, let

$$
K(s, r)=\int_{0}^{1-1 /(r-s)}\left(1-u^{2}\right)^{\delta} u^{j} J_{\alpha}(s u) J_{\beta}(r u) d u .
$$

Then, if either $\beta=\alpha$ and $j$ odd or $\beta=\alpha \pm 1$ and $j$ even, we have,

$$
|K(s, r)| \leq c(r-s)^{-\delta-1}(r(1+s))^{-1 / 2},
$$

with $c$ independent of $r$ and $s$.

PROOF. For each $m \in \mathbf{N}$, we will show that (2.2) holds for $\delta \leq m-2$ by induction on $m$.

Case $m=0$. Since $\alpha \geq 0$ and $\beta \geq 0$, by $(2.1)(\mathrm{c})$

$$
|K(s, r)| \leq c r^{-1 / 2} \int_{0}^{1-1 /(r-s)}\left(1-u^{2}\right)^{\delta} u^{1 / 2}(1+s u)^{-1 / 2} d u .
$$

Now let $s \leq 1$. Then the right side above is bounded by

$$
c r^{-1 / 2} \int_{0}^{1-1 /(r-s)}\left(1-u^{2}\right)^{\delta} d u \leq c r^{-1 / 2}\left((r-s)^{-\delta-1}+1\right) .
$$


But $\delta \leq-2$ as $m=0$ and $r-s \geq 2$, thus,

$$
|K(s, r)| \leq c(r-s)^{-\delta-1} r^{-1 / 2} .
$$

If $s \geq 1$, then from $(2.3)$

$$
\begin{aligned}
|K(s, r)| & \leq c r^{-1 / 2} s^{-1 / 2} \int_{0}^{1-1 /(r-s)}\left(1-u^{2}\right)^{\delta} d u \leq c(r s)^{-1 / 2}\left((r-s)^{-\delta-1}+1\right) \\
& \leq c(r s)^{-1 / 2}(r-s)^{-\delta-1} .
\end{aligned}
$$

Thus combining the two cases $s \leq 1$ and $s \geq 1$, yields that if $\delta \leq-2$, then

$$
|K(s, r)| \leq c(r(1+s))^{-1 / 2}(r-s)^{-\delta-1} .
$$

The step above is the first step in the induction. We now proceed to the induction step itself. Using (2.1)(a), (b), an elementary calculation yields

$$
\begin{aligned}
\frac{d}{d u}\left(u^{j} s J_{\alpha+1}(s u) J_{\beta}(r u)-u^{j} r J_{\alpha}(s u) J_{\beta+1}(r u)\right) \\
=u^{j}\left(s^{2}-r^{2}\right) J_{\alpha}(s u) J_{\beta}(r u)+u^{j-1} s(j-\alpha-1+\beta) J_{\alpha+1}(s u) J_{\beta}(r u) \\
\quad+u^{j-1} r(-j-\alpha+\beta+1) J_{\alpha}(s u) J_{\beta+1}(r u) .
\end{aligned}
$$

Rearranging terms and dividing by $r^{2}-s^{2}$ we have

$$
\begin{aligned}
u^{j} J_{\alpha}(s u) J_{\beta}(r u)= & -\frac{1}{r^{2}-s^{2}} \frac{d}{d u}\left[u^{j} s J_{\alpha+1}(s u) J_{\beta}(r u)-u^{j} r J_{\alpha}(s u) J_{\beta+1}(r u)\right] \\
& +u^{j-1} \frac{s(j-\alpha-1+\beta)}{\left(r^{2}-s^{2}\right)} J_{\alpha+1}(s u) J_{\beta}(r u) \\
& \left.+u^{j-1} \frac{r(-j-\alpha+\beta+1)}{\left(r^{2}-s^{2}\right)} J_{\alpha}(s u) J_{\beta+1}\right)(r u) .
\end{aligned}
$$

We now substitute the right side of the equality above in the integrand for $K(s, r)$. Thus $|K(s, r)|$ is bounded by the sum of the three terms that follow:

$$
\frac{1}{r^{2}-s^{2}}\left|\int_{0}^{1-1 /(r-s)}\left(1-u^{2}\right)^{\delta} \frac{d}{d u}\left(u^{j} s J_{\alpha+1}(s u) J_{\beta}(r u)-u^{j} r J_{\alpha}(s u) J_{\beta+1}(r u)\right) d u\right| \text {, }
$$

$$
\frac{s}{r^{2}-s^{2}}\left|(j-\alpha-1+\beta) \int_{0}^{1-1 /(r-s)}\left(1-u^{2}\right)^{\delta} u^{j-1} J_{\alpha+1}(s u) J_{\beta}(r u) d u\right|
$$

and

$$
\frac{r}{r^{2}-s^{2}}\left|(-j-\alpha+\beta+1) \int_{0}^{1-1(r-s)}\left(1-u^{2}\right)^{\delta} u^{j-1} J_{\alpha}(s u) J_{\beta+1}(r u) d u\right| .
$$

We integrate (2.4) by parts once to get that (2.4) is bounded by the sum of

$$
\begin{aligned}
& \frac{1}{r^{2}-s^{2}}\left|\left(1-u^{2}\right)^{\delta} u^{j}\left(s J_{\alpha+1}(s u) J_{\beta}(r u)-r J_{\alpha}(s u) J_{\beta+1}(r u)\right)\right|_{u=0}^{1-1 /(r-s)} \mid, \\
& \frac{2 s}{r^{2}-s^{2}}\left|\delta \int_{0}^{1-1 /(r-s)}\left(1-u^{2}\right)^{\delta-1} u^{j+1} J_{\alpha+1}(s u) J_{\beta}(r u) d u\right|
\end{aligned}
$$


and

$$
\frac{2 r}{r^{2}-s^{2}}\left|\delta \int_{0}^{1-1 /(r-s)}\left(1-u^{2}\right)^{\delta-1} u^{j+1} J_{\alpha}(s u) J_{\beta+1}(r u) d u\right|
$$

We first estimate (2.7) by employing $(2.1)(\mathrm{c})$. We first note that because $j \geq 1$, the expression (2.7) vanishes at the lower limit. Thus at the upper limit we easily see that $(2.7)$ is bounded by

$$
c(r(1+s))^{-1 / 2} \frac{(r-s)^{-\delta}}{\left(r^{2}-s^{2}\right)}(r+s) \leq c(r(1+s))^{-1 / 2}(r-s)^{-\delta-1},
$$

which is what is required.

We now turn to (2.8) and use the inductive hypothesis. If $\alpha+1=\beta$ or $\alpha=\beta$ the inductive hypothesis applies and (2.8) is bounded by

$$
\frac{c s(r(1+s))^{-1 / 2}}{r^{2}-s^{2}}(r-s)^{-\delta} \leq c(r-s)^{-\delta-1}(r(1+s))^{-1 / 2} .
$$

If $\alpha=\beta+1$ we use the following recurrence formula [11]:

$$
J_{\alpha+1}(s u)=\frac{2 \alpha}{s u} J_{\alpha}(s u)-J_{\alpha-1}(s u) \quad(\alpha \geq 1)
$$

and break up (2.8) into two pieces by substituting the right side of (2.10) for $J_{\alpha+1}(s u)$ in the integrand of (2.8). Thus (2.8) is bounded by

$$
\begin{aligned}
& \frac{2 \alpha}{r^{2}-s^{2}}\left|\delta \int_{0}^{1-1 /(r-s)}\left(1-u^{2}\right)^{\delta-1} u^{j} J_{\alpha}(s u) J_{\beta}(r u) d u\right| \\
& \quad+\frac{2 s}{\left(r^{2}-s^{2}\right)}\left|\delta \int_{0}^{1-1 /(r-s)}\left(1-u^{2}\right)^{\delta-1} u^{j+1} J_{\alpha-1}(s u) J_{\beta}(r u) d u\right| .
\end{aligned}
$$

The induction hypothesis again applies to both pieces, and we again can conclude that $(2.8)$ is bounded by $c(r(1+s))^{-1 / 2}(r-s)^{-\delta-1}$. Note that $\alpha-1=\beta \geq 0$ and so $(2.10)$ is valid.

The analysis of (2.9) is similar. If $\beta+1=\alpha$ or $\beta+1=\alpha+1$, the inductive hypothesis again applies. If $\beta+1=\alpha+2$, then we use (2.10) once more, i.e.,

$$
J_{\beta+1}(r u)=\frac{2 \beta}{r u} J_{\beta}(r u)-J_{\beta-1}(r u) .
$$

Using the above we break up (2.9) into a sum of two pieces analogous to (2.8) and apply the induction hypothesis on each piece to get that $(2.9)$ is bounded by $c(r(1+s))^{-1 / 2}(r-s)^{-\delta-1}$.

We now consider (2.5). If $j=1$, then we have $\alpha=\beta$, and thus $j-\alpha-1+\beta=0$ and (2.5) vanishes. So let $j=2$. We now have two possibilities, either $\beta=\alpha+1$ or $\beta=\alpha-1$. If $\beta=\alpha-1$, then because $j=2, j-a-1+\beta=0$, and again (2.5) vanishes. Thus we are left to handle $j=2$ and $\beta=\alpha+1$. We first write

$$
\left(1-u^{2}\right)^{\delta}=\left(1-u^{2}\right)^{\delta-1}-u^{2}\left(1-u^{2}\right)^{\delta-1}
$$


and use the above to bound (2.5) by the following

$$
\begin{aligned}
& \frac{2 s}{\left(r^{2}-s^{2}\right)}\left|\int_{0}^{1-1 /(r-s)}\left(1-u^{2}\right)^{\delta-1} u J_{\alpha+1}(s u) J_{\beta}(r u) d u\right| \\
& \quad+\frac{2 s}{\left(r^{2}-s^{2}\right)}\left|\int_{0}^{1-1 /(r-s)}\left(1-u^{2}\right)^{\delta-1} u^{3} J_{\alpha+1}(s u) J_{\beta}(r u) d u\right|
\end{aligned}
$$

The induction hypothesis now applies to both pieces and the result follows. We now consider the case $j \geq 3$. So let $j \geq 3$ and let $j$ be odd. Then $\alpha=\beta$ and thus using (2.11) in (2.5), (2.5) is bounded by the sum of the two expressions

$$
\frac{c s}{\left(r^{2}-s^{2}\right)}\left|\int_{0}^{1-1 /(r-s)}\left(1-u^{2}\right)^{\delta-1} u^{j-1} J_{\alpha+1}(s u) J_{\beta}(r u) d u\right|
$$

and

$$
\frac{c s}{\left(r^{2}-s^{2}\right)}\left|\int_{0}^{1-1 /(r-s)}\left(1-u^{2}\right)^{\delta-1} u^{j+1} J_{\alpha+1}(s u) J_{\beta}(r u) d u\right| .
$$

Because $j-1$ and $j+1$ are both even and $\alpha=\beta$, the induction hypothesis applies to both pieces and again (2.5) is bounded by

$$
\frac{c s}{\left(r^{2}-s^{2}\right)}(r(1+s))^{-1 / 2}(r-s)^{-\delta} \leq c(r(1+s))^{-1 / 2}(r-s)^{-\delta-1} .
$$

Thus we are left with considering $j \geq 4$ and $j$ even. Since $j$ is even, $\beta=\alpha \pm 1$. If $\beta=\alpha+1$, then we again use (2.11) to split (2.5) as a sum of two terms, to each of which we apply the inductive hypothesis. If $\beta=\alpha-1$, we apply $(2.10)$ and bound (2.5) by a sum of two pieces,

$$
\begin{aligned}
& \frac{2 \alpha}{\left(r^{2}-s^{2}\right)}\left|(j-\alpha-1+\beta) \int_{0}^{1-1 /(r-s)}\left(1-u^{2}\right)^{\delta} u^{j-2} J_{\alpha}(s u) J_{\beta}(r u) d u\right| \\
& \quad+\frac{s}{\left(r^{2}-s^{2}\right)}\left|(j-\alpha-1+\beta) \int_{0}^{1-1 /(r-s)}\left(1-u^{2}\right)^{\delta} u^{j-1} J_{\alpha-1}(s u) J_{\beta}(r u) d u\right| .
\end{aligned}
$$

Now split each term above into two terms by using (2.11), to get

$$
\begin{aligned}
& \frac{2 \alpha}{\left(r^{2}-s^{2}\right)}\left|(j-\alpha-1+\beta) \int_{0}^{1-1 /(r-s)}\left(1-u^{2}\right)^{\delta-1} u^{j-2} J_{\alpha}(s u) J_{\beta}(r u) d u\right| \\
& +\frac{2 \alpha}{\left(r^{2}-s^{2}\right)}\left|(j-\alpha-1+\beta) \int_{0}^{1-1 /(r-s)}\left(1-u^{2}\right)^{\delta-1} u^{j} J_{\alpha}(s u) J_{\beta}(r u) d u\right| \\
& +\frac{s}{\left(r^{2}-s^{2}\right)}\left|(j-\alpha-1+\beta) \int_{0}^{1-1 /(r-s)}\left(1-u^{2}\right)^{\delta-1} u^{j-1} J_{\alpha-1}(s u) J_{\beta}(r u) d u\right| \\
& +\frac{s}{\left(r^{2}-s^{2}\right)}\left|(j-\alpha-1+\beta) \int_{0}^{1-1 /(r-s)}\left(1-u^{2}\right)^{\delta-1} u^{j+1} J_{\alpha-1}(s u) J_{\beta}(r u) d u\right|
\end{aligned}
$$

The induction hypothesis now applies to each of the four terms, and thus (2.5) is bounded by $c(r(1+s))^{-1 / 2}(r-s)^{-\delta-1}$. 
The estimation of (2.6) proceeds in a manner analogous to that of (2.5) and in fact the necessary modifications are obvious, due to the symmetry in (2.5) and (2.6). We have thus completely proved Lemma (2.2). Q.E.D.

LEMMA (2.12). For $\delta>-1$ and $n \in \mathbf{N}, n \geq 2$, let

$$
k_{\delta}(s, r)=\int_{0}^{1}\left(1-u^{2}\right)^{\delta} u J_{(n-2) / 2}(u r) J_{(n-2) / 2}(u s) d u .
$$

Then, for $|s-r| \geq 2$,

$$
\left|k_{\delta}(s, r)\right| \leq c|r-s|^{-\delta-1}((1+r)(1+s))^{-1 / 2}
$$

PROOF. With no loss of generality assume $r \geq s$. This is because $k_{\delta}(r, s)=$ $k_{\delta}(s, r)$. Moreover because $|r-s|>2$, it follows that $r>\frac{1}{2}$. Thus $(1+r) \sim r$. We split the range of integration to get

$$
\begin{aligned}
k_{\delta}(s, r)= & \int_{0}^{1-1 /(r-s)}\left(1-u^{2}\right)^{\delta} u J_{(n-2) / 2}(u r) J_{(n-2) / 2}(u s) d u \\
& +\int_{1-1 /(r-s)}^{1}\left(1-u^{2}\right)^{\delta} u J_{(n-2) / 2}(u r) J_{(n-2) / 2}(u s) d u
\end{aligned}
$$

Thus,

$$
\left|k_{\delta}(s, r)\right| \leq|K(s, r)|+\left|\int_{1-1 /(r-s)}^{1}\left(1-u^{2}\right)^{\delta} u J_{(n-2) / 2}(u r) J_{(n-2) / 2}(u s) d u\right|
$$

Since $(r-s) \geq 2$, we may apply Lemma (2.2) to the first term on the right above to get

$$
|K(s, r)| \leq c|r-s|^{-\delta-1}(r(1+s))^{-1 / 2} \leq c|r-s|^{-\delta-1}((1+r)(1+s))^{-1 / 2} .
$$

Moreover for $0<u \leq 1$,

$$
(1+u r)^{-1 / 2}(1+u s)^{-1 / 2} \leq u^{-1}(1+r)^{-1 / 2}(1+s)^{-1 / 2} .
$$

Thus by Lemma $(2.1)(\mathrm{c})$,

$$
\begin{aligned}
\left|J_{(n-2) / 2}(u r) J_{(n-2) / 2}(u s)\right| & \leq c(1+u r)^{-1 / 2}(1+u s)^{-1 / 2} \\
& \leq c u^{-1}(1+r)^{-1 / 2}(1+s)^{-1 / 2}
\end{aligned}
$$

Substituting this inequality in the second integrand on the right in (2.13), we get

$$
\begin{aligned}
& \left|\int_{1-1 /(r-s)}^{1}\left(1-u^{2}\right)^{\delta} u J_{(n-2) / 2}(u r) J_{(n-2) / 2}(u s) d u\right| \\
& \quad \leq c(1+r)^{-1 / 2}(1+s)^{-1 / 2} \int_{1-1 /(r-s)}^{1}\left(1-u^{2}\right)^{\delta} d u \\
& \quad \leq c(1+r)^{-1 / 2}(1+s)^{-1 / 2}|r-s|^{-\delta-1} .
\end{aligned}
$$

Thus $c((1+r)(1+s))^{-1 / 2}|r-s|^{-\delta-1}$ bounds both terms on the right in (2.13). This proves the lemma. Q.E.D. 
LEMMA (2.14). Let $k_{\delta}(s, r)$ be defined as in Lemma (2.12). Then for $\delta>-1$,

$$
\left|k_{\delta}(s, r)\right| \leq c((1+r)(1+s))^{-1 / 2} \text {. }
$$

Proof. Using $(2.1)(\mathrm{c})$ in the integral for $k_{\delta}(s, r)$ we see that

$$
\left|k_{\delta}(s, r)\right| \leq c \int_{0}^{1} u\left(1-u^{2}\right)^{\delta}(1+u s)^{-1 / 2}(1+u r)^{-1 / 2} d u \text {. }
$$

Now for $0<u \leq 1,(1+u s)^{-1 / 2}(1+u r)^{-1 / 2} \leq u^{-1}((1+r)(1+s))^{-1 / 2}$. Thus,

$$
\begin{aligned}
\left|k_{\delta}(s, r)\right| & \leq c((1+r)(1+s))^{-1 / 2} \int_{0}^{1}\left(1-u^{2}\right)^{\delta} d u \\
& \leq c((1+r)(1+s))^{-1 / 2} . \quad \text { Q.E.D. }
\end{aligned}
$$

To prove the next lemma, we need to recall that for the Bessel function of order $\alpha \geq 0,\left|J_{\alpha}(x)\right| \leq c x^{\alpha}$ for $x \geq 0$. This may be found in $[\mathbf{1 1}]$.

LEMMA (2.15). Let $k_{\delta}(s, r)$ be defined as in Lemma (2.12). Then for $\delta>-1$,

$$
\left|k_{\delta}(s, r)\right| \leq c(s r)^{(n-2) / 2} .
$$

PROOF. Using the fact that $\left|J_{\alpha}(x)\right| \leq c x^{\alpha}$, it follows that

$$
\left|k_{\delta}(s, r)\right| \leq c(r s)^{(n-2) / 2} \int_{0}^{1}\left(1-u^{2}\right)^{\delta} u^{n-1} d u \leq c(r s)^{(n-2) / 2} \text {. Q.E.D. }
$$

LEMMA (2.16). Let $f$ be supported in $|x| \leq 2$. Then for $|x| \geq 4$,

$$
\left|T_{\delta} f(x)\right| \leq c|x|^{-(n+1+2 \delta) / 2}\|f\|_{p_{0}}, \quad p_{0}=2 n /(n+1+2 \delta) .
$$

PROOF. From $[\mathbf{9}, \text { p. 171 }]^{3}$, it follows that

$$
T_{\delta} f(x)=c_{n} \int_{\mathbf{R}^{n}} f(y) \frac{J_{n / 2+\delta}(|x-y|)}{|x-y|^{n / 2+\delta}} d y .
$$

Thus, from Lemma $(2.1)(\mathrm{c})$,

$$
\left|T_{\delta} f(x)\right| \leq c \int_{|y| \leq 2}|f(y)||x-y|^{-(n+1+2 \delta) / 2} d y .
$$

If $|x| \geq 4$, then $|x-y| \approx|x|$. Thus,

$$
\left|T_{\delta} f(x)\right| \leq c|x|^{-(n+1+2 \delta) / 2} \int_{|y| \leq 2}|f| d y \leq c|x|^{-(n+1+2 \delta) / 2}\|f\|_{p_{0}} \quad \text { Q.E.D. }
$$

3. We now proceed with the proof of Theorem 1 .

Proof of TheOREM 1. We let $s=|x|, r=|y|$ and $t=|\xi|$. By Bochner's formula $[\mathbf{9}$, p. 155],

$$
\hat{f}(\xi)=\hat{f}(t)=c_{n} t^{-(n-2) / 2} \int_{0}^{\infty} f(r) J_{(n-2) / 2}(r t) r^{n / 2} d r .
$$

Moreover,

$$
T_{\delta} f(s)=c_{n} s^{-(n-2) / 2} \int_{0}^{1}\left(1-t^{2}\right)^{\delta} J_{(n-2) / 2}(t s) \hat{f}(t) t^{n / 2} d t .
$$

${ }^{3}$ Note the statement of the Theorem (4.15) has a misprint. 
Substituting the expression for $\hat{f}(t)$ into that for $T_{\delta} f(s)$, we see that

$$
\begin{aligned}
T_{\delta} f(s) & =c_{n}^{2} s^{-(n-2) / 2} \int_{0}^{\infty} f(r) r^{n / 2} \int_{0}^{1} t\left(1-t^{2}\right)^{\delta} J_{(n-2) / 2}(t s) J_{(n-2) / 2}(t r) d t d r \\
& =c_{n}^{2} s^{-(n-2) / 2} \int_{0}^{\infty} f(r) r^{n / 2} k_{\delta}(s, r) d r .
\end{aligned}
$$

We first consider the case $p_{0}>1$, that is $\delta<(n-1) / 2$.

We now consider the situation when $s \leq 4$. We have

$$
\begin{aligned}
\left|T_{\delta} f(s)\right| & \leq c s^{-(n-2) / 2}\left(\int_{0}^{8}|f(r)| r^{n / 2}\left|k_{\delta}(s, r)\right| d r+\int_{8}^{\infty}|f(r)| r^{n / 2}\left|k_{\delta}(s, r)\right| d r\right) \\
& =c s^{-(n-2) / 2}(A+B) .
\end{aligned}
$$

By Lemma (2.15) when $s \leq 4$ and $r<8,\left|k_{\delta}(s, r)\right| \leq c(r s)^{(n-2) / 2}$. Thus,

$$
s^{-(n-2) / 2} A \leq c \int_{0}^{8}|f(r)| r^{n-1} d r \leq c\|f\|_{p_{0}} .
$$

Since $s \leq 4$, we have

$$
s^{-(n-2) / 2} A \leq c s^{-(n+1+2 \delta) / 2}\|f\|_{p_{0}} .
$$

Now consider $s^{-(n-2) / 2} B$. Since $s \leq 4$ and $r>8, r>2 s$, and thus by Lemma (2.12), with $1 / p_{0}+1 / q_{0}=1$, we have

$$
\begin{aligned}
s^{-(n-2) / 2} B \leq & c s^{-(n-2) / 2} \int_{8}^{\infty}|f(r)| r^{(n-1) / 2-\delta-1} d r \\
\leq & c s^{-(n-2) / 2}\left(\int_{0}^{\infty}|f(r)|^{p_{0}} r^{n-1} d r\right)^{1 / p_{0}} \\
& \cdot\left(\int_{1}^{\infty} r^{-(1+\delta+(n-1) / 2) q_{0}+n-1} d r\right)^{1 / q_{0}} \\
\leq & c s^{-(n+1+2 \delta) / 2}\|f\|_{p_{0}} .
\end{aligned}
$$

Thus for $s \leq 4$,

$$
\left|T_{\delta} f(s)\right| \leq c s^{-(n+1+2 \delta) / 2}\|f\|_{p_{0}} .
$$

We now pass to the case $s>4$. We make a preliminary reduction. Let $f(r)=$ $f_{1}(r)+f_{2}(r)$, where $f_{1}(r)=f(r) \chi(r \leq 2)$. Then, by Lemma (2.16),

$$
\left|T_{\delta} f_{1}(s)\right| \leq c s^{-(n+1+2 \delta) / 2}\left\|f_{1}\right\|_{p_{0}} \leq c s^{-(n+1+2 \delta) / 2}\|f\|_{p_{0}} .
$$

We now estimate $T_{\delta} f_{2}(s)$. We then have

$$
\begin{gathered}
\left|T_{\delta} f_{2}(s)\right| \leq c s^{-(n-2) / 2}\left(\int_{2}^{s / 2}+\int_{s / 2}^{2 s}+\int_{2 s}^{\infty}\left|f_{2}(r)\right| r^{n / 2}\left|k_{\delta}(s, r)\right| d r\right) \\
=c s^{-(n-2) / 2}(D+E+F) .
\end{gathered}
$$


By Lemma (2.12) if $r<s / 2,\left|k_{\delta}(s, r)\right| \leq s^{-\delta-3 / 2} r^{-1 / 2}$. Thus, with $1 / p_{0}+1 / q_{0}=1$, we have,

$$
\begin{aligned}
s^{-(n-2) / 2} D & \leq c s^{-(n+1+2 \delta) / 2} \int_{2}^{s / 2}|f(r)| r^{(n-1) / 2} d r \\
& \leq c s^{-(n+1+2 \delta) / 2}\|f\|_{p_{0}}\left(\int_{2}^{\infty} r^{-q_{0}(n-1) / 2+n-1} d r\right)^{1 / q_{0}} \\
& \leq c s^{-(n+1+2 \delta) / 2}\|f\|_{p_{0}} .
\end{aligned}
$$

For the integral $F$, since $r>2 s,\left|k_{\delta}(s, r)\right| \leq c r^{-\delta-1}(r s)^{-1 / 2}$. Thus with $1 / p_{0}+$ $1 / q_{0}=1$,

$$
\begin{aligned}
s^{-(n-2) / 2} F & \leq c s^{-(n-1) / 2} \int_{2 s}^{\infty}|f(r)| r^{n / 2-\delta-3 / 2} d r \\
& \leq c s^{-(n-1) / 2}\|f\|_{p_{0}}\left(\int_{2 s}^{\infty} r^{-(n+1+2 \delta) q_{0} / 2+n-1} d r\right)^{1 / q_{0}} \\
& \leq c s^{-(n+1+2 \delta) / 2}\|f\|_{p_{0}}, \quad \text { since } s>1 .
\end{aligned}
$$

We now consider $s^{-(n-2) / 2} E$. We first break up the range of integration for $E$ as follows:

$$
\begin{aligned}
s^{-(n-2) / 2} E \leq & s^{-(n-2) / 2} \int_{\{s / 2<r<2 s\} \cap\{|r-s|>2\}}|f(r)| r^{n / 2}\left|k_{\delta}(s, r)\right| d r \\
& +s^{-(n-2) / 2} \int_{\{s / 2<r<2 s\} \cap\{|r-s| \leq 2\}}|f(r)| r^{n / 2}\left|k_{\delta}(s, r)\right| d r .
\end{aligned}
$$

By Lemma (2.14)

$$
\begin{aligned}
& s^{-(n-2) / 2} \int_{\{s / 2<r<2 s\} \cap\{|r-s|<2\}}|f(r)| r^{n / 2}\left|k_{\delta}(s, r)\right| d r \\
& \leq c \int_{|r-s|<2}|f(r)| \chi\left(\frac{s}{2}<r<2 s\right) d r \\
& \leq c\left(f \chi_{H_{s}}\right)^{*}(s),
\end{aligned}
$$

where $H_{s}=\{r: s / 2<r<2 s\}$. Moreover, by Lemma (2.12)

$$
\begin{aligned}
& s^{-(n-2) / 2} \int_{H_{s} \cap\{|r-s|>2\}}|f(r)| r^{n / 2}\left|k_{\delta}(s, r)\right| d r \\
& \leq c s^{-(n-1) / 2} \int_{H_{s} \cap\{|r-s|>2\}}|f(r)||r-s|^{-\delta-1} r^{(n-1) / 2} d r \\
& \quad \leq \int_{|r-s|>2}|f(r)| \chi_{H_{s}}(r)|r-s|^{-\delta-1} d r \\
& \quad=\sum_{k \geq 0} 2^{-k \delta}\left(2^{-k} \int_{|r-s| \sim 2^{k}}|f(r)| \chi_{H_{s}}(r) d r\right) \\
& \leq c\left(f \chi_{H_{s}}\right)^{*}(s) .
\end{aligned}
$$

Thus,

$$
s^{-(n-2) / 2} E \leq c\left(f \chi_{H_{s}}\right)^{*}(s)
$$


Collecting the estimates (3.1)-(3.5), it follows that

$$
\left|T_{\delta} f(s)\right| \leq c s^{-(n+1+2 \delta) / 2}\|f\|_{p_{0}}+c\left(f \chi_{H_{s}}\right)^{*}(s) .
$$

Thus, letting $I_{k}=\left\{s: 2^{k-1} \leq s<2^{k}\right\}$ and $H_{k}=\left\{r: 2^{k-2} \leq r<2^{k+1}\right\}$, we have,

$$
\begin{aligned}
\int_{\left\{s: T_{\delta} f(s) \mid>\lambda\right\}} s^{n-1} d s \leq & \int_{\left\{s: s^{-(n+1+2 \delta) / 2}\|f\|_{p_{0}}>\lambda / 2 c\right\}} s^{n-1} d s \\
& +\int_{\left\{s:\left(f \chi_{H_{s}}\right)^{*}(s)>\lambda / 2 c\right\}} s^{n-1} d s \\
\leq & c \lambda^{-p_{0}}\|f\|_{p_{0}}^{p_{0}}+\sum_{k=-\infty}^{\infty} \int_{\left\{s \in I_{k},\left(f \chi_{H_{k}}\right)^{*}(s)>\lambda / 2 c\right\}} s^{n-1} d s \\
\leq & c \lambda^{-p_{0}}\|f\|_{p_{0}}^{p_{0}}+\sum_{k=-\infty}^{\infty} 2^{k(n-1)} \int_{\left\{s \in I_{k},\left(f \chi_{H_{k}}\right)^{*}(s)>\lambda / 2 c\right\}} d s .
\end{aligned}
$$

By the weak $\left(p_{0}, p_{0}\right)$ estimate for the Hardy-Littlewood maximal function (note $p_{0}=1$ is possible) we can majorize the expression above by

$$
c \lambda^{-p_{0}}\left(\|f\|_{p_{0}}^{p_{0}}+\sum_{k=-\infty}^{\infty} 2^{k(n-1)} \int_{H_{k}}|f|^{p_{0}} d r\right) .
$$

Thus by bounded overlaps.

$$
c \lambda^{-p_{0}}\left(\|f\|_{p_{0}}^{p_{0}}+\sum_{k=-\infty}^{\infty} 2^{k(n-1)} \int_{H_{k}}|f(r)|^{p_{0}} d r\right) \leq c \lambda^{-p_{0}}\|f\|_{p_{0}}^{p_{0}} .
$$

We are now left to consider the case $p_{0}=1$ or $\delta=(n-1) / 2$. The only expressions we treat differently for this case are the integrals $B, D$ and $F$. The other parts are the same as for the case $p_{0}>1$. Thus if $\delta=(n-1) / 2$,

$$
\begin{aligned}
s^{-(n-2) / 2} B & \leq c s^{-(n-2) / 2} \int_{8}^{\infty}|f(r)| r^{-1} d r \\
& \leq c s^{-n} \int_{8}^{\infty}|f(r)| r^{n-1} d r \leq c s^{-n}\|f\|_{1} .
\end{aligned}
$$

Likewise,

$$
\begin{aligned}
s^{-(n-2) / 2} D & \leq c s^{-n} \int_{1}^{s / 2}|f(r)| r^{(n-1) / 2} d r \\
& \leq c s^{-n} \int_{1}^{\infty}|f(r)| r^{n-1} d r \leq c s^{-n}\|f\|_{1} .
\end{aligned}
$$

Now,

$$
\begin{aligned}
s^{-(n-2) / 2} F & \leq c s^{-(n-1) / 2} \int_{2 s}^{\infty}|f(r)| r^{-1} d r \\
& \leq c s^{-(n-1) / 2}\left(\int_{2 s}^{\infty}|f(r)| r^{n-1} d r\right) s^{-n}
\end{aligned}
$$

Recalling that $s \geq 4$, it follows that

$$
s^{-(n-2) / 2} F \leq c s^{-n}\|f\|_{1}
$$

We may now proceed as in the case $p_{0}>1$. This completes the proof of the theorem. Q.E.D. 


\section{REFERENCES}

1. A. P. Calderón and A. Zygmund, On singular integrals, Amer. J. Math. 78 (1956), 289-309.

2. S. Chanillo, The multiplier for the ball and radial functions, J. Funct. Anal. 55 (1984), 18-24.

3. A. Cordoba, A note on Bochner-Riesz operators, Duke Math. J. 46 (1979), 505-511.

4. C. Fefferman, Inequalities for strongly singular convolution operators, Acta Math. 124 (1970), 9-36.

5. __ The multiplier problem for the ball, Ann. of Math. (2) 94 (1971), 330-336.

6. C. Herz, On the mean inversion of Fourier and Hankel transforms, Proc. Nat. Acad. Sci. U.S.A. 40 (1954), 996-999.

7. C. Kenig and P. Tomas, The weak behavior of spherical means, Proc. Amer. Math. Soc. 78 (1980), 48-50.

8. E. Stein, Interpolation of linear operators, Trans. Amer. Math. Soc. 87 (1958), 159-172.

9. E. Stein and G. Weiss, Introduction to Fourier analysis on Euclidean spaces, Princeton Univ. Press, Princeton, N.J., 1971.

10. P. Tomas, A restriction theorem for Fourier transforms, Bull. Amer. Math. Soc. 81 (1975), 477-478.

11. G. N. Watson, A treatise on the theory of Bessel functions, 2nd ed., Cambridge Univ. Press, London and New York, 1966.

Department of Mathematics, institute for AdVAnCed Study, Princeton, NEW JERSEY 08540

Department of MAThematics, Rutgers University, NEW BRUNSWiCK, NeW JeRSEY 08903 (Current address of Benjamin Muckenhoupt)

Current address (S. Chanillo): Department of Mathematics, Ohio State University, Columbus, Ohio 43210 